\title{
Investigating risks of outcome-based service contracts from a provider's perspective
}

\begin{abstract}
Outcome-based contracts (OBC) refer to agreements between providers and customers where providers are paid based on the outcomes they deliver to customers. OBCs have become common for manufacturing firms that are servitizing, especially those firms providing advanced services focusing on availability and capability. While many authors acknowledge the importance of understanding risks in OBCs from a provider's perspective, there are relatively few in depth extant studies. To address this gap in the literature, we conducted 24 interviews with 11 companies to explore risks and risk factors in OBCs from the provider's perspective. The case studies reveal that the two major risk categories in OBCs are commercial risk regarding the contract negotiations and contract decisions at the contracting stage, and operational risk regarding the implementation and the delivery of OBCs. Our study identifies 23 risk factors that can lead to commercial risk and operational risk in five dimensions: i) complexity and ii) dynamism regarding the context of OBCs, and iii) capability, iv) alignment and v) dependency regarding the stakeholders of OBCs. The paper explores the links between these risk factors and commercial and operational risk. In doing so the paper provides a framework for understanding risk in OBCs.
\end{abstract}

Keywords: risk management, risk analysis, performance-based contracting, product service systems, outcome-based contract, servitization

\section{Introduction}

It is clear that significant changes are occurring in manufacturing industries. Many firms - particularly those involve in supplying complex capital equipment - are innovating their business models and focusing on the provision of services and solutions, rather than just the equipment (Neely 2009, Baines et al. 2009). Authors refer to this phenomenon as servitization, which has been defined as "the innovation of organisation's capabilities and processes to better create mutual value through a shift from selling products to selling Product-Service Systems” (Neely 2008). Studies have highlighted different forms of servitization. A useful classification is provided by Baines and Lightfoot (Baines and Lightfoot 2013) who distinguish between basic services such as after-sale service, to intermediate services such as condition monitoring, to advanced services where contracts are struck on the basis of availability and outcomes. Different forms of commercial arrangements and contracting often accompany these different forms of services. Basic services are often accompanied by contracts that cover inputs - traditional Time and Material (T\&M) contracts. Intermediate services often include service level agreements (SLA) which focus on the outputs a customer receives. While advanced 
services focus on outcomes - often gain share and pain share mechanisms that are incorporated into outcome-based contracts (OBC) or performance-based contracts (PBC).

This study focuses specifically on outcome-based contracts (OBC). The recent systematic literature review of OBCs/PBCs shows a clear increasing interest in the topic (Selviaridis and Wynstra 2015). While the terms have been used widely in different contexts with different meanings, such as the payment scheme for executives to incentivize their performance, it is pointed out that "PBC is highly relevant in the context of servitisation of manufacturing” (Selviaridis and Wynstra 2015). There have been many successful examples of OBCs, or PBCs in the servitization context, such as Rolls-Royce's “Power-by-the-Hour”, and its developed versions of CorporateCare ${ }^{\circledR}$ and TotalCare ${ }^{\circledR}$ in the defence and corporate sectors and the civil aviation sector respectively. Joining the two highly relevant research areas of servitization and OBC, we focus on outcome-based contract in the servitization context in this paper.

The adoption of new pricing policies can increase risks for servitizing firms (Cavalieri and Pezzotta 2012). This is especially the case for the adoption of OBCs. From being contracted on inputs or outputs, to being contracted on outcomes, providers usually take on increased risks (Roehrich and Caldwell 2012, Hypko, Tilebein, and Gleich 2010). In T\&M contracts, providers are paid based on consumed resources and take few risks. When SLAs are adopted, providers are paid for service delivered often to pre-agreed service levels. Hence providers face risks associated with their failure to deliver efficiently to the agreed service levels. In OBCs, however, more risks are introduced to providers for three reasons. First, the high complexity of delivering total solutions leads to higher risks. Second, in many cases, providers don't have full control of the outcomes, which are affected by many external factors such as customers' behaviours. One example was given by Ng et al. (Ng, Maull, and Yip 2009), "a pilot that is more careful about the use of the equipment such as 'taking care when removing the communication plug' instead of carelessly and unknowingly flinging it, and hitting the windscreen (resulting in chipping glass) can save the firm £18,000 per piece of glass”. Third, by providing OBCs, providers usually take on responsibilities that were previously taken on by their customers. Risks are transferred to the providers along with these responsibilities.

In this situation, a study on risks of OBCs from the provider's perspective is important, and risk has identified to be one of the three key dimensions of OBCs in the literature, together with performance and incentives (Selviaridis and Wynstra 2015). However, there are still limited systematic studies revealing risks and their formations in OBCs. Answering the call for more research on risks (Selviaridis and Wynstra 2015), this paper aims to fill this research gap by investigating risks of OBCs from a provider's perspective. The structure of this paper is as follows: in section two, we review extant literature on risks of OBCs. After that, the research design is introduced in section three, 
followed by the main findings in section four and discussions in section five. In section six, conclusions and future research suggestions are presented.

\section{Literature Review}

As discussed, the research presented in the paper focuses on exploring the risks associated with OBCs from a provider's perspective. To fulfill this purpose, we will first define OBCs and risk, then introduce what risks have been identified in the literature of OBCs.

Outcome-based contracts (OBC), or in other names, performance-based contracts, performance-based contracting, performance-based logistics, have been defined in the literature variously. They refer to contracts where customers focus on or pay for outcomes of equipment (Ng and Yip 2009), the accomplishment of performance goals (Hypko, Tilebein, and Gleich 2010) or outcomes of customer value (Guajardo et al. 2012, Kleemann and Essig 2013, Kim, Cohen, and Netessine 2007) instead of activities, tasks (Ng and Yip 2009), behaviours (Hypko, Tilebein, and Gleich 2010), individual components of a solution (Kleemann and Essig 2013), a set of prescribed specifications (Bramwell 2003), or how to achieve the performance goals (Hypko, Tilebein, and Gleich 2010). In this research, we define an outcome-based contract as "an agreement between the provider and the customer that the provider provides total solutions and is paid based on the outcomes of the solutions or the outcomes of customer value in a continual use situation". We emphasize on three important elements of OBCs. First, in OBCs, total solutions are to be delivered instead of individual components such as pure products or individual service activities, and the contracts specify outcomes instead of inputs, processes or outputs. Second, the payments the provider receives depend on the outcomes of the total solutions or the outcomes of customer value. In the former situation, the outcomes or performances of what is delivered are contracted for. For example, the availability contracts in the defence industry where the provider has the responsibility to ensure the availability of aircrafts to be at a certain level and to carry out all related services are OBCs. In the latter situation, what the customer achieves in its own businesses with the total solutions delivered by the provider are contracted for. For example, in the education industry, the contracts where service providers provide education solutions to colleges and are contracted for the pass rates or the graduation rates of the college students are OBCs. Third, the agreements should be in a continual use situation, referring to the relational instead of transactional nature of the relationship between the provider and the customer in OBCs.

The definition of risk also varies in different contexts. In this study, based on the definition by Hopkin (Hopkin 2012), we define risk as "an event with the ability to directly inhibit the mission, strategy, projects, routine operations, objectives, core processes, key dependencies and/or the delivery of stakeholder expectations". Because the research on risks of OBCs is still an emerging field, this research focuses on the risk identification of OBCs. The different models for risk identification in the literature of risk management (Hallikas, Virolainen, and Tuominen 2002, Ishikawa 1988, Hopkin 
2012) imply a common understanding that risk events and the causal factors should be identified separately, so the mechanism how risk factors lead to the happening of risks can be unveiled and understood. Following their proposals, we aim to identify risks and risk factors of OBCs. Risks, as defined, are events with the ability to directly inhibit the objectives, while risk factors are the status or situations that can lead to the happening of risks.

Though still emerging, risks of OBCs for providers are discussed in the literature (Hypko, Tilebein, and Gleich 2010, Ng, Maull, and Yip 2009, Erkoyuncu, Roy, and Shehab 2014). Extant literature does not distinguish risks and risk factors, but analyzing the literature in this way, we are able to better understand the literature, identify gaps and position our research. The literature review shows that two risks are discussed in the literature: (1) unsuitable contract decisions and (2) difficulties or failure of providers to deliver services effectively. Regarding the first risk, unsuitable contract decisions can be made by the providers, such as unsuitable KPIs and incomplete contracts (Fearnely, Bekken, and Norheim 2004, Hensher and Stanley 2010, Erkoyuncu, Roy, and Shehab 2014). Without suitable KPIs to measure outcomes, the contracts cannot drive the preferred behaviours (Fearnely, Bekken, and Norheim 2004) and the customers cannot receive desired services. Lack of clarity in the contracts is common when delivering complex services with OBCs, resulting in high transaction costs during service delivery (Hensher and Stanley 2010). Regarding the second risk, service providers find it challenging to deliver services and to fulfill their obligations effectively (Ng, Maull, and Yip 2009).

Examining risk factors that lead to the happening of risks, especially the risk of "difficulties or failure of providers to deliver services effectively”, the literature has identified eight risk factors. (1) Service providers play in a complex environment. Applying OBCs, they will have to compete with more experienced service providers (Raddats and Easingwood 2010), or even their own customers who traditionally conducted some services themselves (Hypko, Tilebein, and Gleich 2010), increasing the difficulties and complexity in the service delivery. (2) The environment where service providers deliver OBCs is dynamic. External factors such as the overall economic situations, the technology development, etc. introduce high dynamism and uncertainty that providers cannot control by their behaviours (Hypko, Tilebein, and Gleich 2010). (3) Customer demands are dynamic due to reasons such as their own performances in the market, the shift in their manufacturing plans, etc. (Hypko, Tilebein, and Gleich 2010), which increases the difficulties for the service providers to fulfill customer demands. (4) Some providers lack capabilities to deliver OBCs, such as service operation capabilities (Raddats and Easingwood 2010), knowledge and expertise, financial capabilities (Hypko, Tilebein, and Gleich 2010), human resources (Ng, Maull, and Yip 2009), etc. (5) Providers can experience internal resistance towards the adoption of OBCs and conflicts can arise between the service divisions and the product divisions (Raddats and Easingwood 2010). (6) Customers' failure in fulfilling their responsibilities, such as using the machinery inappropriately, causes additional maintenance costs to the providers (Hypko, Tilebein, and Gleich 2010). (7) Mismatching in 
understandings between the providers and the customers exists. Providers are not fully aware of the usage of the equipment, the customers' operation behaviours, the potential technological changes of the customers, or the customers' value propositions (Ng, Maull, and Yip 2009). So it is difficult for providers to deliver required services effectively and to predict costs in advance. (8) Upfront investments are usually required from providers of OBCs. Providers may retain the ownership of machinery or equipment and deliver outcomes or performances to customers. With such big investments, they will have high dependency on the customers and on third parties such as financial institutes (Hypko, Tilebein, and Gleich 2010).

Table 1 summarizes risks and risk factors of OBCs identified in the literature.

(Table 1 in the end of the manuscript)

The literature review enables us to identify two research gaps in the current literature. First, a systematic research on risks of OBCs is still required to obtain a relatively complete list of risks and risk factors. The risks and risk factors summarized in Table 1 scatter in the literature with limited explanations, and whether key risks and risk factors have been identified is then suspicious. For example, while the long-term duration is an important characteristic of OBCs (Sols, Nowich, and Verma 2007), it has not been discussed as a risk factor. Furthermore, no framework has been proposed regarding risks of OBCs. A framework is important to lay a concrete foundation for future research on this topic and to provide practitioners a way of considering risks of OBCs. Second, how risk factors contribute to the happening of risks has not been well explained and requires further exploration. So in this paper, we aim to fill the research gaps and to explore risks associated with OBCs from a provider's perspective.

\section{Research Design}

Case studies were conducted to answer the research question for three reasons (Voss, Tsikriktsis, and Frohlich 2002, Yin 2013). First, as introduced in the literature review, the research on risks of OBCs from the provider's perspective is still limited. To study this innovative area where limited knowledge has been accumulated, qualitative research is suitable. Second, to identify what the risks of OBCs are and why they exist requires a close examination of the risks in OBCs, and involves the interviewees' personal experience. Case studies can lead to deep insights. Third, this research aims to build a framework regarding risks of OBCs, which has a theory-building purpose.

We use both secondary interview data and primary interview data to understand risks of OBCs. Before we went to the field and conducted interviews, we first analysed a group of secondary interviews conducted by other service researchers to provide guidance for the primary data collection and data analysis. In total, nine secondary interviews from three companies were analysed. Company A is a world leading producer of aero engines; Company B is a major player in the defence industry 
and Company $\mathrm{C}$ is a publishing and education company. All these companies are known for their outcome-based contracting models to deliver services and solutions.

The secondary data reveals that risks can rise both at the contract negotiation stage and at the contract implementation stage, which is in line with the literature where risks of unsuitable contract decisions and difficulties or failure of providers to deliver services effectively are identified. So we decided to discuss risks and risk factors of $\mathrm{OBC}$ at the contract negotiation stage and at the contract implementation stage respectively in the primary data collection.

During the primary data collection, we conducted 24 semi-structured interviews with 11 companies. The unit of analysis is outcome-based service contracts. The focal companies are pioneering in servitization, and interviewees were or are involved in the negotiation and / or implementation of outcome-based service contracts. At the beginning of the interviews, interviewees were asked to describe the OBCs they are / were involved in, such as the provided products and services, the pricing model, etc. Their descriptions were compared with our definition of OBCs, and only those that met our definition were kept for data analysis. To ensure the generalizability of the research, these companies cover a wide range of industries, such as industrial equipment manufacturing and services, media, IT, telecommunications, aerospace and defence, air transport communications, etc., and interviewees cover a wide range of roles in the organizations from account managers to executives.

An interview invitation letter describing the research topic and the research background was first sent to potential interviewees, followed by the research outline and the research questions. The research protocol was designed based on the current research, and risks of OBCs at the negotiation stage and at the implementation stage were explored respectively. Semi-structured interviews were conducted with open questions to explore the topic. The research questions were non-leading questions to ensure objectivity, and interviewees were asked to explain the risks of OBCs that they encountered or were concerned with. After that, factors or reasons why these risks existed were discussed. All interviews were recorded with permission, and were transcribed word by word for data analysis. Risks and risk factors mentioned in the interviews were then identified and coded. When interviewees wanted to review the findings, case reports were produced and asked for feedbacks. The research topic and the findings were discussed widely with different groups of people and audience over the past five years.

\section{Findings from the case studies}

\subsection{Risks and risk factors of $O B C$}

The analysis of the secondary data leads us to the finding that risks rise both at the contracting stage and at the delivery stage, and we refer to the former as commercial risk and the latter as operational risk. Commercial risk refers to the risk regarding the contract negotiations and contract decisions at the contracting stage, such as unsuitable contract decisions - overpromise in the agreements, unsuitable payment mechanism, unsuitable KPIs, lack of clarity in the contracts, etc., or complex 
contract negotiations - the negotiation process is complex, time-consuming and costly. Operational risk refers to the risk regarding the implementation and the delivery of OBCs, such as complexity in service delivery, providers' failing to deliver required services, customers' failing to achieve expected value, etc.

We then analyse the primary data, aiming to identify risk factors that can lead to the happening of commercial risk and/or operational risk. Twenty-three risk factors that can lead to commercial risk and/or operational risk are identified, shown in Table 2. We will explain each risk factor in detail what it means and how it leads to commercial and/or operational risk.

(Table 2 in the end of the manuscript)

\section{Involvement of multiple stakeholders}

Increasingly OBCs involve multiple stakeholders at different stages of the contracts. The focal provider collaborates with other companies to deliver advanced services and total solutions. Besides, different departments within the provider's and the customer's organizations are involved. Stakeholders could also be end users, third parties, any other organizations or individuals that can influence the contracting and the delivery of OBCs. All stakeholders have their own priorities, interests and drivers, which are different or even in tension. Negotiating with stakeholders on key topics such as products and services to be included in the total solutions, accountability and rewards of each stakeholder, KPIs, etc. can be extremely complex. This risk factor also contributes to the complexity in implementation. Providers find it difficult to manage the contract and to manage the outcomes, and they will never foresee where risks will come from during the implementation. When risks happen, the unclear accountability can lead to the situations where stakeholders criticize each other and shirk their responsibilities. So the involvement of multiple stakeholders leads to both commercial and operational risk.

\section{Diversified customer demands}

Country and regional differences are important reasons why customers have diversified demands. Customers from different countries will have different cultures and understandings of OBCs. For example, customers in some countries want very complete contracts with all details to be included, while customers in other countries are more interested in the general principles behind the details. Even within one country, customer requirements in different regions can be very different due to their diversified business situations and business drivers. The diversified customer demands mean customized contracts and increasing complexity of the negotiation process. The complexity is further 
increased by the fact that some international customers increasingly require global integrations within their various business locations, giving challenges to the provider when contracting OBCs.

\section{Unclear customer demands}

In many cases, the outcome-based model is new to the customer, and it is difficult for the customer to clearly define its requirements. The lack of clarity will make the negotiation process challenging and complex. One senior account executive said in the interview, "there was risk with the customer in the sense that they didn't know where to start, like piling more and more things into the bucket that they wanted. It took 18 months to go from a kind of initial conceptual discussions to signing the agreement". The unclear customer demands can also result in delays in the service delivery, or reduced customer satisfaction. The customer will be disappointed and the provider will face financial loss or reputational damage.

\section{Complex contracts}

Compared with traditional contracts, OBCs can be very complex, involving many variables that need to be considered when the contracts are established. Also the provider and the customer need to consider how these variables will be affected by external factors such as commodity prices, overall economic situations, etc., how each variable may change, and how the changes may influence the outcomes. With such complex contracts, it is challenging for the provider to fully understand the consequences of their decisions, and to measure the impacts of potential risks. So the provider can make unsuitable decisions in the contracts, resulting in commercial risk.

\section{Complex environment}

The overall complexity in economy, industry and society should also be considered by service providers, as they can lead to both commercial and operational risk. For example, in a highly competitive industry where other providers play aggressively to enter the service business, the focal provider may give up more when negotiating the contracts. And in an industry which is strictly regulated or is shrinking overall, it is challenging for the provider to scale up or to retain scales. The natural and social environment where the services and solutions are delivered can be complex as well, with many situations unforeseeable, increasing the provider's risks during delivery.

\section{Dynamic customer demands}

Customers will have their own drivers for the contracts and these drivers can be very fluid, resulting in dynamic customer demands. Without realizing and preventing the potential dynamism in the contract, the provider may be in high risk when the delivery sets off. Besides, if the customer lacks the capability to consume provided services, or if customer demands are not specified clearly during the 
negotiation, it can constantly change the details of their requirements during implementation. As a result, "there is a change, there is extra time, extra effort, extra costs that need to be spent". "Dynamic customer demands" differs from "unclear customer demands" in the sense that "unclear customer demands" refers to the situations where customers do not know what they want, or only know their demands in general, mainly due to the lack of experience in OBCs; while "dynamic customer demands" refers to the situations where customers know what they want or what they don't want, and constantly change those demands due to internal or external reasons. Unclear customer demands during negotiation could lead to dynamic customer demands during implementation.

\section{Dynamic environment}

When providers negotiate OBCs, they will calculate variables based on particular weights, such as the labour price, the oil price, the inflation, etc. However, these variables may change over time during implementation, and providers may not have the opportunity to renegotiate the contracts, resulting in financial loss. For providers in specific industries or working closely with the government, the dynamism introduced by politics can be very high, as described by one interviewee that "one thing I see from working with the military customer is that a full load is nowhere nearing the predictable... so that sort of unpredictability to the demand means that high priced risks to the contract becomes very difficult”. So contracting and delivering OBCs in a dynamic environment is challenging, and commercial and operational risk can happen due to the dynamism.

\section{Long-term contracts}

Highly linked with the risk factor of dynamic environment is the fact that OBCs are usually long-term contracts lasting for years. The variables that were calculated at the negotiation may not be suitable any more, as well as other decisions the providers made in the contracts. Combined with the risk factor that some providers lack capabilities to establish OBCs, and do not build in flexibility in the contracts, providers can make unsuitable decisions in the contracts, and suffer during the entire period of the contract delivery. An example was given in the interview, "a bit of business that was very profitable is now a problem. But how do you judge three years out, what the world can look like in three years". As discussed in the factor of "complex environment", the provider may give up more at the negotiation if the industry is highly competitive, and it has to bear the results for relatively long term. Renegotiation is not always an option and the provider may suffer from financial loss.

\section{Providers' lack of capabilities to contract OBC}

When moving to OBCs, many providers have not acquired enough experience, knowledge and capabilities for contracting outcomes. They are not aware of the best practices to follow at the negotiation and the potential consequences of their decisions, nor do they have enough data and 
information to support their decisions on some of the most important issues such as pricing model, KPIs, service solutions, cost analysis and risk assessment, etc. One pricing manager who led the negotiations of OBCs said in the interview, "we don't have a standard process and we don't have the core competence. So what ends up happening is we end up getting a team together for every single one we do. None of us have detailed training on drafting these agreements." In this situation, the provider can make unsuitable decisions in the contract which turn out to be highly risky during the implementation. For example, some of the KPIs that were put in place to measure outcomes do not necessarily mean that they are good for value achievement for the customer.

\section{Providers' lack of capabilities to deliver OBC}

The provider's lack of capability to deliver service is an important reason of operational risk that the provider fails to deliver services. There are different capabilities that are required for service delivery with OBCs, such as the project management capability, the expertise and resource capability, the supply chain management, the service and products design, data management, service delivery, technological capability, etc. One interviewee gave an example that "the upcoming stages will involve much more sophisticated levels of technology on our side and on theirs. If our products cannot meet those requirements, the risk is that we will ultimately not hit the delivery in the next steps of capability they are asking for." So the provider's lack of capabilities to deliver outcome-based agreements leads to operational risk during contract implementation.

\section{Providers' internal inconsistency}

Another aspect of the provider's lack of capabilities is internal inconsistency, referring to three types of inconsistency: (1) longitudinal inconsistency between the negotiation and the implementation teams, (2) hierarchical inconsistency between the senior leadership and the project levels, and (3) horizontal inconsistency among different departments within the provider's organization. Overpromise is a common consequence of internal inconsistency. In some cases, people who will implement the contract are not involved in the negotiation of the contract. In other situations, the senior leadership makes imprudent decisions, as said in one of the interviews, "they (senior leadership) can overcommit...you can end up with a disconnection between the senior leadership and the working level. Then there's a very real risk”. Apart from the commercial risk of unsuitable contract decisions, the provider's lack of internal consistency can lead to complexity in implementation, thus operational risk. For example, account managers may have to bear risks they don't want to bear because they were not involved when the agreements were made; the customer feels confused to receive different messages from different departments of the provider; the turnover of staff increases the challenges to maintain the level of performance, etc. 


\section{Providers' internal resistance}

Internal resistance is another issue reflecting the provider's lack of capabilities. There are several reasons why internal resistance exists. The first reason is that making the shift to OBCs involves many changes in the provider's organization, and people may not be willing to make the changes, with concerns of additional work, potential failure, or the loss of control. Second, in many cases when the solutions to be provided incorporate products or services from different divisions or departments of the provider, the negotiation will become extremely complex regarding the decisions on the offerings, the pricing, etc. Internal conflicts can rise due to political reasons, where different departments fight for their own performance, resources and influences. Third, in some companies, even the top management level is embracing the idea of OBCs, many middle level managers and local franchises insist on traditional contracts to sell pure products and refuse the entire concept of servitization, creating the "permafrost” for transformation. Internal resistance increases the complexity and difficulties at all stages of OBC, resulting in both commercial and operational risk.

\section{Customers' lack of capabilities to consume the delivery and to play their roles}

The customers' lack of capabilities to consume provided service or to play their roles can lead to operational risk that services are not delivered successfully or customers cannot get expected value. If the customer's business processes cannot consume the provided solutions and services, the customer continually imposes changes on the provider, making the delivery complex and challenging. Besides, to ensure the successful delivery, there are tasks that should be undertaken by the customer, and the customer may fail to play its roles. For example, when service providers provide education services and solutions, failure can be simply due to the customer colleges' inability to recruit enough qualified students in the program.

\section{Other stakeholders' lack of capabilities to perform}

The involvement of multiple stakeholders has been identified as a risk factor. All the stakeholders have their roles to fulfill to ensure the success of OBCs. However, some stakeholders do not have enough capabilities to perform, resulting in operational risk of deliver failure. For example, a case company providing energy service in India relies on the grid and national power supply, however, "the whole grid is right at the limit, they had a massive outage last year, where half of North India was just out for days, because the whole thing collapsed."

\section{Mismatching in goals between providers and customers}

The goals of the provider and the customer could be mismatching both in the short term and in the long term. In the short term, the solutions and outcomes that are proposed by the provider and expected by the customer can be very different, and both parties need to make efforts to achieve 
alignments. In the long term, one of the many reasons that the customer requires OBCs is stability. However, from the provider's perspective, they would like to have a certain level of flexibility, considering the fact that OBCs usually last for years and building in no flexibility in the contract is highly risky. One pricing manager said in the interview, "what we find is that they (customers) want stability, they want to know in three years what's going to happen, and we know we don't know, so it's a risk planning, and that's what can cause most of the arguments in these outcome-based decisions". Apart from the commercial risk, operational risk can also happen at the delivery that the provider may realize that the real goals of the customer are different from what it proposed, increasing the complexity of the service delivery.

\section{Mismatching in visions between providers and customers}

When the provider is a pioneer in providing services by OBCs, some of its customers may refuse to change their minds and make the shift, saying that "we've always done our maintenance, we are in control, we don't want to give you control, we feel we are losing something", so the negotiation becomes very difficult. Lack of alignment in visions also means that the provider and the customer have different visions regarding the nature of OBCs and nature of the relationships. From some providers' perspective, the importance of OBCs is to set practical ground rules for implementations and to build a collaborative relationship. While some customers still think in a traditional way, considering the contracts as rigid agreements without flexibility. In that sense, every time when a problem rises, the provider thinks to work together with the customer to solve the problem, while the customer thinks to go back to the contract and ends up in a contract review. When customers are not fully into the concept of OBCs, they don't consider the provider as a partner and don't work together with the provider with openness and in the spirit of partnership. So gaps in visions on servitization, on the nature of OBCs and on the nature of the relationships lead to both commercial risk at the contracting stage and operational risk at the implementation stage.

\section{Mismatching in practices between providers and customers}

Mismatching in practices of negotiating and implementing OBCs between the provider and the customer can lead to commercial risk and operational risk. At the negotiation stage, the customer will have their own financial modelling and indexes they want to use, and the practices they want to follow, which are different from the provider in some cases. At the implementation stage, the customer may want to be involved in the micro-management while the provider needs freedom for decision making with least interventions. Efficiency will be reduced and arguments may rise due to the gaps in practices. 


\section{Mismatching in understandings between providers and customers}

In some cases the provider and the customer lack mutual understandings. When the provider traditionally provides products and is still strong in product provisions, people will either not realize it is a service and solution provider, or the image of a product provider is still deep in people's minds. It leads to complexity in negotiation and the provider needs to explain to the customer who they are and what they do, and the initial approach to the customer is not easy due to the lack of mutual understandings. Besides, without a deep understandings of the customer, the provider will sign the contract without fully realizing the possible consequences. At the contract implementation stage, there can be gaps in understandings of each other's business processes, resulting in complexity, confusion and even delivery failure.

\section{Mismatching in culture between providers and customers}

Culture differences can make the implementation of the contract complex and difficult. Cultural differences are from many aspects, such as the provider's and the customer's communication styles, working styles, leaderships, etc. Culture differences are prominent if the provider and the customer are different types of organizations in nature - one in the private sector and one in the public sector, or if they are from completely different cultural backgrounds. Gaps in culture can lead to difficulties in communications and lack of collaboration. In that situation, failure to deliver the required services and solutions can happen.

\section{Mismatching in bargaining power between providers and customers}

Power imbalance can exist that the provider does not have equal bargaining power with the customer. For example, when the provider lacks the capability or experience to negotiate OBCs, customers will take advantage of that and come to the negotiation better prepared. In other situations, the provider is not confident enough to provide OBCs due to lack of experience, or the provider still has the subordinate mentality in the relationship. As a result, the provider loses initiatives and has to follow what the customer proposes or to compromise during the negotiation. Also, the provider may be required to provide core and detailed information without getting key information back from the customer, though this information may largely influence the outcomes. Power imbalance can also lead to the provider's failure in delivering required services if the customer steps in inappropriately during implementation.

\section{Dependency on customers}

The service delivery requires customers' inputs in different ways, from information, infrastructure, to skilled labour, or parts of the total solutions, etc. However, the providers' influences on customers' performances and behaviours are limited, as said in the interview, "outcome of performance is 
dependent on availability of people on the customer side and on our side...those are the things that we don't fully control, and therefore it's more difficult for us to manage, but we are trying obviously". The provider bears the consequences of things they don't have full control of, which is an obvious risk factor during implementation. Besides, the customers' failure in their own businesses can lead to payment delays, or even the termination of contracts.

\section{Dependency on other stakeholders}

Similarly, the delivery of total solutions requires inputs from different partners and stakeholders, and the provider's dependency on these stakeholders varies to different extent, and bears different consequences if the stakeholders fail to play their roles, such as delivery failure, additional costs, the loss of customers, etc.

\section{Upfront investments}

The outcome nature of the contracts sometimes means that the supplier needs to make preinvestments before the client will start to realize the benefits and the value. A manager said in the interview, "there might be cases where there might be more investment upfront or the costs upfront incurred by the service provider. But that's the nature of an outcome-based contract because from the investment perspective, the service provider needs to invest upfront a lot more money before they start seeing the payment or start earning back the investment that they made in an outcome-based contract”, which indicates the potential risks of financial loss if outcomes are not delivered.

\subsection{The risk framework of $\mathrm{OBC}$}

In section 4.1, we have explained the twenty-three risk factors we identified from the case studies, as well as how each of them contributes to commercial risk at the contracting stage and / or operational risk at the implementation stage. Based on the twenty-three risk factors, we will build a framework on risks of OBCs. A close examination of the twenty-three risk factors reveals that these factors can be categorized into five dimensions, which are complexity, dynamism, capability, alignment and dependency. Complexity and dynamism are regarding the context where OBCs are delivered, and capability, alignment and dependency are regarding the stakeholders that are involved in the OBCs.

Complexity: the context where OBCs are adopted is very complex in general, where the providers are exposed to a lot of variables and uncertainty in many aspects. Five risk factors are categorized in this dimension: involvement of multiple stakeholders, diversified customer demands, unclear customer demands, complex contracts, and complex environment. Without realizing, analysing and reducing the context complexity, providers of OBCs can suffer from commercial risk and operational risk.

Dynamism: in parallel with the dimension of complexity which describes the current context of an OBC, another context factor is dynamism, which considers the timeline and reveals the fact that 
OBCs are usually long-term contracts, and dynamism and fluidity are unavoidable. The three risk factors in this dimension are dynamic customer demands, dynamic environment, and long-term contracts. The dynamism can lead to both commercial and operational risk.

Capability: lack of capabilities from key stakeholders - the provider, the customer, other partners, etc. is an important reason why commercial risk and operational risk happen. In this dimension, we categorize six risk factors that contribute to commercial risk and operational risk, which are (1) providers' lack of capabilities to contract OBC, (2) providers' lack of capabilities to deliver OBC, (3) providers' internal inconsistency, (4) providers' internal resistance, (5) customers' lack of capabilities to consume the delivery and to play their roles, and (6) other stakeholders' lack of capabilities to perform.

Alignment: mismatching between the provider and the customer is identified to be an important dimension of risk factors why commercial risk and operational risk happen. Alignment between the provider and the customer needs to be achieved in six aspects, which are goals, visions, practices, understandings, culture and bargaining power.

Dependency: this dimension emphasizes first, the extent to which the provider depends on customers and other stakeholders for the delivery of the services and solutions; second, the extent to which the provider can control or influence the performances and behaviours of customers and other stakeholders; and third, the severity of consequences and impacts the provider has to bear due to the failure of customers and other stakeholders. Upfront investments, which is an important characteristic of OBCs, can also increase the provider's dependency, especially when the investments are contract specific.

Based on the five dimensions, the risk framework of OBC is proposed in Figure 1. It reveals that five dimensions of risk factors are complexity, dynamism as context factors, and capability, alignment and dependency as stakeholder factors. Commercial risk and operational risk are the two broad categories of risks of OBC. The former refers to risks regarding the contract negotiations and contract decisions, while the latter refers to risks regarding the contract implementation and delivery.

(Figure 1 in a separate file)

In this paper, we also try to provide practical suggestions on risk management for practitioners of OBCs. In Table 3, we analyse the contributions of each risk factor to commercial risk and operational risk respectively. Though further quantitative study is required, the number in Table 3 shows how many interviewees identify a risk factor to be the reason of commercial risk or operational risk. For commercial risk, the most discussed risk factors are involvement of multiple stakeholders, providers' 
lack of capabilities to contract OBC, diversified customer demands, providers' internal inconsistency and long-term contracts. For operational risk, the most discussed risk factors are providers' lack of capabilities to deliver OBC, customers' lack of capabilities to consume the delivery and to play their roles, involvement of multiple stakeholders, providers' internal inconsistency, and other stakeholders' lack of capabilities to perform. Adding the contributions of risk factors to commercial risk and operational risk, total contributions reveal that involvement of multiple stakeholders, providers' lack of capabilities to deliver OBC and providers' internal inconsistency should be paid high attention to in risk management of OBC.

(Table 3 in the end of the manuscript)

\section{Discussions}

While outcome-based contracts or performance-based contracts are becoming prominent for many servitizing firms, the definition of OBC still lacks clarity. In this research, we define an outcomebased contract as "an agreement between the provider and the customer that the provider provides total solutions and is paid based on the outcomes of the solutions or the outcomes of customer value in a continual use situation”. Researchers have called for more research exploring risks of OBCs from the provider's perspective, because the risk transfer to the provider is a key feature of this type of contracts (Selviaridis and Wynstra 2015). This paper focuses on exploring risks of OBC from a provider's perspective in the context of servitization. In particular, we are seeking to: (1) identify the range of risks providers might face with OBCs, and (2) identify the reasons why risks emerge.

Our research design and case selection criteria ensure that this research is in the context of servitization and focuses on outcome-based contracts that meet our definition: the provision of total solutions, the payment based on outcomes and the continual use situation. Though increasingly the provision of advanced services adopts OBCs or PBCs, it should be noted that there is not one-to-one correspondence between the two. While advanced services refer to certain types of services provided by servitizing firms, OBCs or PBCs refer to certain types of revenue models which could be used by advanced services, as well as other types of services provided by servitizing firms. The research results are relevant in the context of servitization where OBCs are used.

The key contributions we are making are first, we differentiate commercial risk at the contracting stage and operational risk at the delivery stage. Second, we identify twenty-three risk factors, and explain how these risk factors lead to commercial risk and / or operational risk. Third, we categorize risk factors in five dimensions and propose a risk framework of OBCs. Fourth, we evaluate the contributions of each risk factor to commercial risk and operational risk respectively. In the following 
paragraph, we emphasize some of the key theoretical and practical implications, and compare the findings with previous research.

Summarily, all risks and risk factors discussed in the literature have been verified in this research, but we enrich the discussions and supplement the current research with a more complete list. Some of the identified risk factors have important practical implications. First, "involvement of multiple stakeholders" has been identified in this research to be highly accountable for both commercial and operational risk. Service providers should take key stakeholders into consideration at all stages of the contracts and balance their interests and priorities. Second, the long-term characteristic of OBCs is not emphasized as an important risk factor in the literature. In the case studies, however, practitioners are concerned with the fact that most OBCs last for years, and it is difficult to predict what can happen within years when the contracts are negotiated. A long-term contract could be beneficial as long as flexibility is built in. Here "flexibility" reveals an important issue related with the long-term characteristic of OBCs, which firms using OBCs should be aware of and apply in the contracts where possible. Third, we emphasize that "providers' lack of capabilities” includes not only the lack of capabilities to deliver OBCs as discussed in the literature, but also the lack of capabilities to negotiate and to establish OBCs, as well as the lack of organizational capabilities such as the internal inconsistency. Practitioners should enhance their capabilities and experiences of negotiating OBCs to reduce risks. Fourth, the literature does not pay much attention to the mismatching between the providers and the customers in OBCs, and its potential contributions to risks. In this research, we identify the mismatching between the providers and the customers from six aspects - goals, visions, practices, understandings, culture and bargaining power. Practitioners should devote to reduce the gaps in these aspects and achieve alignments with the customers to reduce commercial and operational risk. Fifth, we propose that the providers' dependency on customers and other stakeholders can increase risks. Understanding the dependency can facilitate providers to better understand potential risks they take in OBCs.

In the risk framework of OBC shown in Figure 1, we summarize the key findings from the research and make contributions theoretically and practically. Theoretically, it proposes a framework summarizing risks and risk factors of $\mathrm{OBC}$, instead of individual risks and risk factors scattered in the literature. The framework lays a foundation for future research, where researchers can further explore each dimension in detail and study how and to what extent it influences risks taken by providers. Opportunities also exist to explore how these dimensions influence each other. Practically, it provides practitioners a neat way of thinking risks of OBCs. They will need to understand the complexity and dynamism of the context where OBCs are applied, and examine the capabilities, alignment and dependency of stakeholders involved in the contracts, both at the contracting stage and at the implementation stage. Starting from this conceptual overview, practitioners can then probe into each 
dimension and evaluate the risk factors based on their situations. Table 3 is useful by providing an initial evaluation of the contributions of each risk factor to commercial and operational risk.

\section{Conclusions}

In this paper, we explore risks associated with OBCs from a provider's perspective in the context of servitization. The provision of advanced services increasingly adopts OBCs to incorporate gain share / pain share mechanisms, where service providers bear high risks. To supplement the current research on risks of OBCs, we conducted case studies with servitizing firms which adopt OBCs to deliver total solutions, and identified twenty-three risk factors in OBCs. These twenty-three risk factors can be categorized into five dimensions - complexity, dynamism, capability, alignment and dependency. The former two are regarding the context of OBCs, while the latter three are regarding the stakeholders of OBCs. We differentiate commercial risk at the contract negotiation stage and operational risk at the contract implementation stage, and evaluate the contributions of each risk factor to commercial risk and operational risk respectively. By proposing the risk framework of OBCs which summarizes risks and risk factors, we contribute to the current research on risks of OBCs in the servitization context. The framework provides opportunities for future research, and also provides guidance for risk management for practitioners who adopt OBCs in their servitization journey.

There are three streams of future research that can be conducted following this research. First, to further understand the contributions to commercial risk and operational risk by the risk factors in five dimensions, to evaluate the importance of risk factors, and to understand the potential interactions among these risk factors, a quantitative research is suggested. Second, this research does not study longitudinally the servitization process and the development of OBCs in the case firms. The interviews reveal that some firms are more experienced than others in providing OBCs, and firms' level of maturity in providing OBCs may affect risks. A longitudinal study exploring the changes of provider risks in OBCs is suggested. Third, this research explores risks of OBC from a provider's perspective, but does not compare provider risks in traditional contracts and in OBCs, nor does it compare provider risks and customer risks in OBCs. So it does not reveal how risk is transferred from the customer to the provider in OBCs. These two comparisons are suggested to unveil the risk transfer phenomenon and mechanism in OBCs.

\section{Reference}

Baines, T. S., H. W. Lightfoot, O. Benedettini, and J. M. Kay. 2009. "The servitization of manufacturing: A review of literature and reflection on future challenges." Journal of Manufacturing Technology Management no. 20 (5):547-567. doi: 10.1108/17410380910960984.

Baines, Tim, and Howard Lightfoot. 2013. Made to Serve: How Manufacturers Can Compete Through Servitization and Product Service Systems. United Kingdom: John Wiley \& Sons Ltd.

Bramwell, J. 2003. What is performance based building? In Performance-based Building, First International State of the Art Report, edited by A. Lee and Barrett.

Cavalieri, Sergio, and Giuditta Pezzotta. 2012. "Product-Service Systems Engineering: State of the Art and Research Challenges." Computers in Industry no. 63 (4):278-288. 
Erkoyuncu, John Ahmet, Rajkumar Roy, and Essam Shehab. 2014. "An innovative uncertainty management framework to support contracting for product-service availability." Journal of Service Management no. 25 (5):603-638.

Fearnely, Nils, Jon-Terje Bekken, and Bard Norheim. 2004. "Optimal performance-based subsidies in Norwegian intercity rail transport." International Journal of Transport Management no. 2:2938.

Guajardo, J. A., M. A. Cohen, S. H. Kim, and S. Netessine. 2012. "Impact of Performance-Based Contracting on Product Reliability: An Empirical Analysis." Management Science no. 58 (5):961-979. doi: 10.1287/mnsc.1110.1465.

Hallikas, Jukka, Veli-Matti Virolainen, and Markku Tuominen. 2002. "Risk analysis and assessment in network environments: A dyadic case study." Int. J.Production Economics no. 78.

Hensher, David A., and John Stanley. 2010. "Contracting regimes for bus services: What have we learnt after 20 years?" Research in Transportation Economics no. 29 (1):140-144.

Hopkin, Paul. 2012. Fundamentals of risk management: understanding evaluating and implementing effective risk management. Second ed. United Kingdom: Kogan Page Limited.

Hypko, Phillipp, Meike Tilebein, and Ronald Gleich. 2010. "Benefits and uncertainties of performance-based contracting in manufacturing industries: An agency theory perspective." Journal of Service Management no. 21 (4):460-489. doi: 10.1108/09564231011066114.

Ishikawa, K. et al. 1988. What is Total Quality Control? Englewood Cliffs, NJ: Prentice Hall.

Kim, Sang-Hyun, Morris A. Cohen, and Serguei Netessine. 2007. "Performance Contracting in AfterSales Service Supply Chains." Management Science no. 53 (12):1843-1858.

Kleemann, Florian C., and Michael Essig. 2013. "A providers' perspective on supplier relationships in performance-based contracting." Journal of Purchasing and Supply Management.

Neely, Andy. 2008. The servitization of Manufacturing: An Analysis of Global Trends. In The 14th European Operation Management Association Conference. Ankara, Turkey.

Neely, Andy. 2009. "Exploring the financial consequences of the servitization of manufacturing." Operations Management Research no. 1 (2):103-118. doi: 10.1007/s12063-009-0015-5.

$\mathrm{Ng}$, Irene C. L. , and Nick Yip. 2009. Identifying Risk and its Impact on Contracting Through a Benefit Based-Model Framework in Business to Business contracting: Case of the defence industry. In CIRP Design Conference, edited by R. Rajkumar. Cranfield, U.K.

$\mathrm{Ng}$, Irene C. L., Roger Maull, and Nick Yip. 2009. "Outcome-based contracts as a driver for systems thinking and service-dominant logic in service science: Evidence from the defence industry." European Management Journal no. 27 (6):377-387. doi: 10.1016/j.emj.2009.05.002.

Raddats, Chris, and Chris Easingwood. 2010. "Services growth options for B2B product-centric businesses." Industrial Marketing Management no. 39 (8):1334-1345. doi: 10.1016/j.indmarman.2010.03.002.

Roehrich, J.K, and N.D Caldwell. 2012. "Delivering integrated solutions in the public sector: The unbundling paradox." Industrial Marketing Management no. 41 (6):995-1007.

Selviaridis, Kostas, and Finn Wynstra. 2015. "Performance-based contracting: a literature review and future research directions." International Journal of Production Research no. 53 (12):35053540.

Sols, Alberto, David Nowich, and Dinesh Verma. 2007. "Defining the Fundamental Framework of an Effective Performance-Based Logistics (PBL) Contract." Engineering Management Journal no. 19 (2):40-50.

Voss, Chris, Nikos Tsikriktsis, and Mark Frohlich. 2002. "Case Research in Operations Management." International Journal of Operations \& Production Management no. 22 (2):195-219.

Yin, Rober K. 2013. Case Study Research: Design and Methods, Applied Social Research Methods: SAGE Publications. 
Table 1 Risks and risk factors of OBC from a provider's perspective in the literature

\begin{tabular}{|c|}
\hline Risks of $\mathbf{O B C}$ in the literature \\
\hline Unsuitable contract decisions \\
Difficulties or failure of providers to \\
deliver services effectively
\end{tabular}

\begin{tabular}{|l|}
\hline \multicolumn{1}{|c|}{ Risk factors of OBCs in the literatures } \\
\hline Complex environment \\
\hline Dynamic environment \\
\hline Dynamic customer demands \\
\hline Providers' lack of capabilities to deliver OBC \\
\hline Providers' internal resistance \\
\hline Customers' lack of capabilities to play their roles \\
\hline $\begin{array}{l}\text { Mismatching in understandings between the } \\
\text { providers and the customers }\end{array}$ \\
\hline Upfront investments \\
\hline
\end{tabular}


Table 2 Risks and risk factors of OBC from a provider's perspective identified from the case studies

\begin{tabular}{|c|c|}
\hline $\begin{array}{l}\text { Risks of OBC from } \\
\text { case studies }\end{array}$ & Risk factors of OBC from case studies \\
\hline \multirow{11}{*}{$\begin{array}{l}\text { Commercial risk } \\
\text { regarding the contract } \\
\text { negotiations and } \\
\text { contract decisions, e.g. } \\
\text { unsuitable contract } \\
\text { decisions, complex } \\
\text { negotiation process }\end{array}$} & Involvement of multiple stakeholders \\
\hline & Diversified customer demands \\
\hline & Unclear customer demands \\
\hline & Complex contracts \\
\hline & Complex environment \\
\hline & Dynamic customer demands \\
\hline & Dynamic environment \\
\hline & Long-term contracts \\
\hline & Providers' lack of capabilities to contract OBC \\
\hline & Providers' lack of capabilities to deliver OBC \\
\hline & Providers’ internal inconsistency \\
\hline \multirow{12}{*}{$\begin{array}{l}\text { Operational risk } \\
\text { regarding the contract } \\
\text { implementation and } \\
\text { delivery, e.g. } \\
\text { complexity in service } \\
\text { delivery, failure to } \\
\text { achieve customer value }\end{array}$} & Providers' internal resistance \\
\hline & $\begin{array}{l}\text { Customers' lack of capabilities to consume the delivery and to play } \\
\text { their roles }\end{array}$ \\
\hline & Other stakeholders' lack of capabilities to perform \\
\hline & Mismatching in goals between providers and customers \\
\hline & Mismatching in visions between providers and customers \\
\hline & Mismatching in practices between providers and customers \\
\hline & Mismatching in understandings between providers and customers \\
\hline & Mismatching in culture between providers and customers \\
\hline & Mismatching in bargaining power between providers and customers \\
\hline & Dependency on customers \\
\hline & Dependency on other stakeholders \\
\hline & Upfront investments \\
\hline
\end{tabular}


Table 3 Contributions of risk factors to commercial risk and operational risk (by number of interviews)

\begin{tabular}{|c|c|c|c|c|}
\hline Dimension & $\begin{array}{c}\text { Risk factors of OBC from case } \\
\text { studies }\end{array}$ & $\begin{array}{c}\text { Commercial } \\
\text { risk }\end{array}$ & $\begin{array}{l}\text { Operational } \\
\text { risk }\end{array}$ & $\begin{array}{c}\text { Total } \\
\text { contribution }\end{array}$ \\
\hline \multicolumn{5}{|c|}{ Context factors } \\
\hline \multirow{5}{*}{ Complexity } & Involvement of multiple stakeholders & 7 & 6 & 13 \\
\hline & Diversified customer demands & 5 & 0 & 5 \\
\hline & Unclear customer demands & 3 & 1 & 4 \\
\hline & Complex contracts & 3 & 0 & 3 \\
\hline & Complex environment & 1 & 4 & 5 \\
\hline \multirow{3}{*}{ Dynamism } & Dynamic customer demands & 2 & 3 & 5 \\
\hline & Dynamic environment & 1 & 4 & 5 \\
\hline & Long-term contracts & 4 & 1 & 5 \\
\hline \multicolumn{5}{|c|}{ Stakeholder factors } \\
\hline \multirow{6}{*}{ Capability } & $\begin{array}{l}\text { Providers' lack of capabilities to } \\
\text { contract OBC }\end{array}$ & 7 & 0 & 7 \\
\hline & $\begin{array}{l}\text { Providers' lack of capabilities to deliver } \\
\text { OBC }\end{array}$ & 1 & 14 & 15 \\
\hline & Providers’ internal inconsistency & 5 & 6 & 11 \\
\hline & Providers’ internal resistance & 2 & 2 & 4 \\
\hline & $\begin{array}{l}\text { Customers' lack of capabilities to } \\
\text { consume the delivery and to play their } \\
\text { roles }\end{array}$ & 0 & 7 & 7 \\
\hline & $\begin{array}{l}\text { Other stakeholders' lack of capabilities } \\
\text { to perform }\end{array}$ & 0 & 6 & 6 \\
\hline \multirow{6}{*}{ Alignment } & $\begin{array}{l}\text { Mismatching in goals between } \\
\text { providers and customers }\end{array}$ & 3 & 1 & 4 \\
\hline & $\begin{array}{l}\text { Mismatching in visions between } \\
\text { providers and customers }\end{array}$ & 1 & 3 & 4 \\
\hline & $\begin{array}{l}\text { Mismatching in practices between } \\
\text { providers and customers }\end{array}$ & 2 & 1 & 3 \\
\hline & $\begin{array}{l}\text { Mismatching in understandings between } \\
\text { providers and customers }\end{array}$ & 3 & 3 & 6 \\
\hline & $\begin{array}{l}\text { Mismatching in culture between } \\
\text { providers and customers }\end{array}$ & 0 & 2 & 2 \\
\hline & $\begin{array}{l}\text { Mismatching in bargaining power } \\
\text { between providers and customers }\end{array}$ & 4 & 1 & 5 \\
\hline \multirow{3}{*}{ Dependency } & Dependency on customers & 0 & 10 & 10 \\
\hline & Dependency on other stakeholders & 0 & 7 & 7 \\
\hline & Upfront investments & 1 & 4 & 5 \\
\hline
\end{tabular}




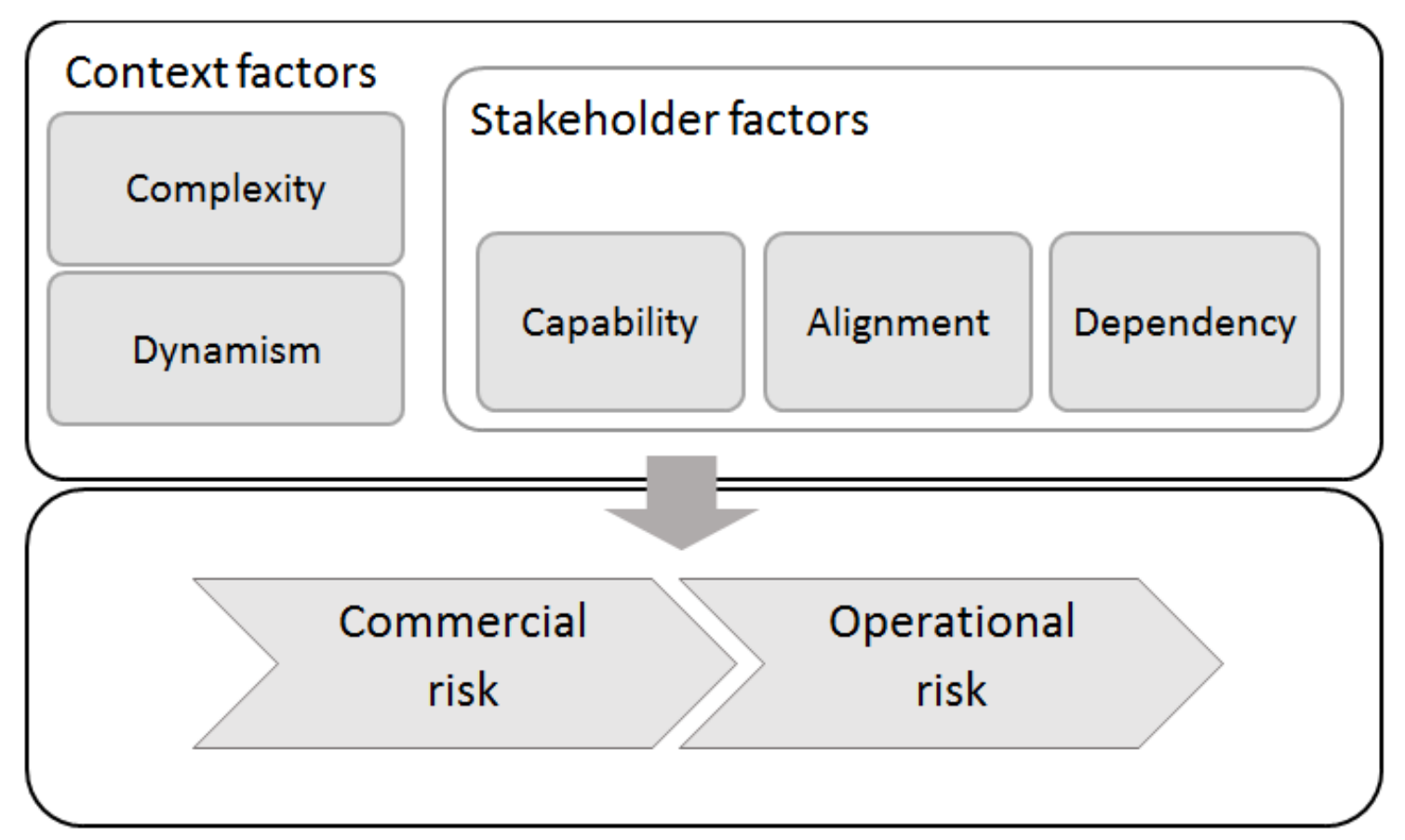

Figure 1 Risk framework of outcome-based contracts from a provider's perspective 